\title{
Melt Polycondensation Process of Poly(Ethylene Terephthalate) in Grid Falling Film Tower
}

\author{
Zhenhao XI ${ }^{1}$, Ling $\mathrm{ZHAO}^{1}$, Weizhen SUN ${ }^{1}$ \\ and Zhaoyan $\mathrm{LIU}^{2}$ \\ ${ }^{1}$ State Key Laboratory of Chemical Engineering, East China \\ University of Science and Technology, Shanghai 200237, P.R. China \\ ${ }^{2}$ Beijing Innovation Polymerization Technology Company, \\ Beijing 100050, P.R. China
}

Keywords: Polycondensation, Poly(Ethylene Terephthalate), Grid Falling Film Tower, Surface Renewal, Model

\begin{abstract}
Melt polycondensation processes to manufacture poly(ethylene terephthalate) with high molecular weight have been realized successfully in a novel reactor, grid falling film tower, in which polymer melt flows through multi-layers grids from top to bottom to form falling film owing to gravity and large gas-liquid interfacial area is generated stably and uniformly by the support of combs. The surface velocity field on falling film was measured to characterize surface renewal of falling film. Little back-mixing exists in down flowing stream, and the fluid flow in GFFT behaves as plug flow. A model has been proposed for continuous PET melt polycondensation process in grid falling film tower and verified by pilot-scale experimental data. Calculated degree of polymerization axial distribution can provide some valuable information for how to match the core structure with the progress of PET polycondensation process.
\end{abstract}

\section{Introduction}

Since poly(ethylene terephthalate) (PET) is a commodity product with a wide applications, ranging from the production of synthetic fibers, films, bottles to engineering plastics, it is of great practical interest to optimize reactor design and enhance PET synthesis. Polycondensation processes are frequently equilibrium limited and thus the condensation by-products should be removed in time to achieve desired molecular weight. Reaction and mass transfer are coupled in PET polycondensation process. As the viscosity of the polymer melt typically increases by orders of magnitude with the reaction proceeding, the removal of the small molecules becomes more and more difficult, and the polycondensation process becomes gradually limited by mass transfer (Rieckmann and Völker, 2001). For PET melt polycondensation process, the removal of the condensation by-products becomes very important after the degree of polymerization (DP) is beyond 30 (Ravindranath and Mashelkar, 1984). Therefore, the design and operation of a polycondensation reactor are usually focused on intensifying the mass transfer. A variety of agitator and reactor configurations for the PET melt polycondensation can be found in the patent literature (Kilpatrick, 1966; Ogata et al., 1969; Hachiya et al., 1970; Crawford et al., 1971). The industrial PET melt polycondensation reactor usually consists

Received on July 12, 2008; accepted on December 17, 2008. Correspondence concerning this article should be addressed to $\mathrm{Z}$. Xi (E-mail address: zhaoling@ecust.edu.cn).

Presented at ISCRE 20 in Kyoto, September, 2008. of a horizontal cylindrical vessel with a rotating shaft on which circular discs, cages, or shallow flight screws are mounted. A horizontal reactor is partially filled with polymer melt and can pick up the melt spreading in form of a thin film over a large area of the discs. In the use of such reactors for the production of particularly viscous polycondensates, difficulties arise because viscous materials do not drop readily enough from the wall, discs and the shaft, and then some special baffle elements and scraping elements have to be added (Lester and Grifon, 1970; Wilhelm et al., 1991). For PET melt polycondensation in horizontal cylindrical vessel, a part of melts are at the bottom of reactor with $0.1-0.2$ times of reactor diameter level, the hydrostatic head keeps accordingly, which has negative effect on the reversible polycondensation reaction. Around $8-10 \%$ dead zone also exists, which would be likely to result in PET degradation. Apart from these particular agitating elements to yield large interfacial area in a horizontal cylindrical vessel, fluid flowing down along special supports to form liquid films, filaments and columns in a vertical vessel can also provide a great lot of sites for devolatilization (Yokoyama and Fujimoto, 2005).

The grid falling film tower (GFFT) (Liu, 1998) has been invented as a novel polycondensation reactor for high viscous fluid. In this reactor, low molecular weight pre-polymer can be supplied in and the melt flows through multi-layers grids from top to bottom to form falling film owing to gravity without agitation, large gas-liquid interfacial area is generated for the removal of volatile by-products, the grids are perpendicular between 


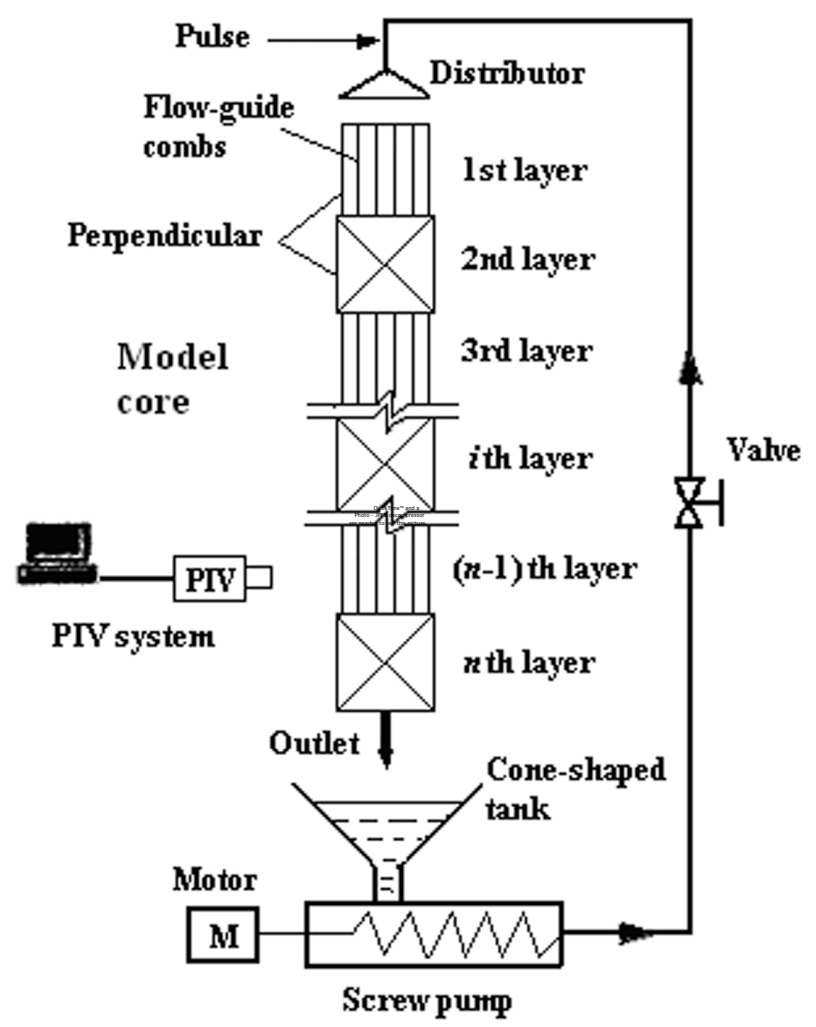

Fig. 1 Equipments for model experiments (The model core is $100 \times 100 \mathrm{~mm}$ )

adjacent layers to ensure essential film renewal and to achieve uniformly flowing. After flowing through the last layer, polymer melt fall down to a receiving funnel, and are pumped out subsequently. The volatile by-products diffuse out of falling film surface, go along the paths among films and gather, then flow upward and are drawn out of the reactor. In our early work (Xi et al., 2007), the configuration of GFFT had been described in detail and the performance of film forming had been studied in the range of fluid viscosity with 1.5-470 Pa.s. It was found that thin falling film could be generated stably and uniformly by the support of combs, and the specific interfacial area of GFFT could reach over $200 \mathrm{~m}^{2} / \mathrm{m}^{3}$. It was also testified that the GFFT have capability to realize PET melt polycondensation and obtain PET product with high molecular weight. In this paper, for the purpose of optimizing reactor construction as well as PET polycondensation in GFFT, the characteristics of falling film and fluid flow in GFFT are further studied, and a model has been developed for PET melt polycondensation process in GFFT to simulate the axial DP distribution.

\section{Experimental}

The model equipments (Figure 1) were built to investigate the characteristics of falling film and fluid flow in GFFT, which were carried out in a controlled atmosphere and measurements were made in the steady state

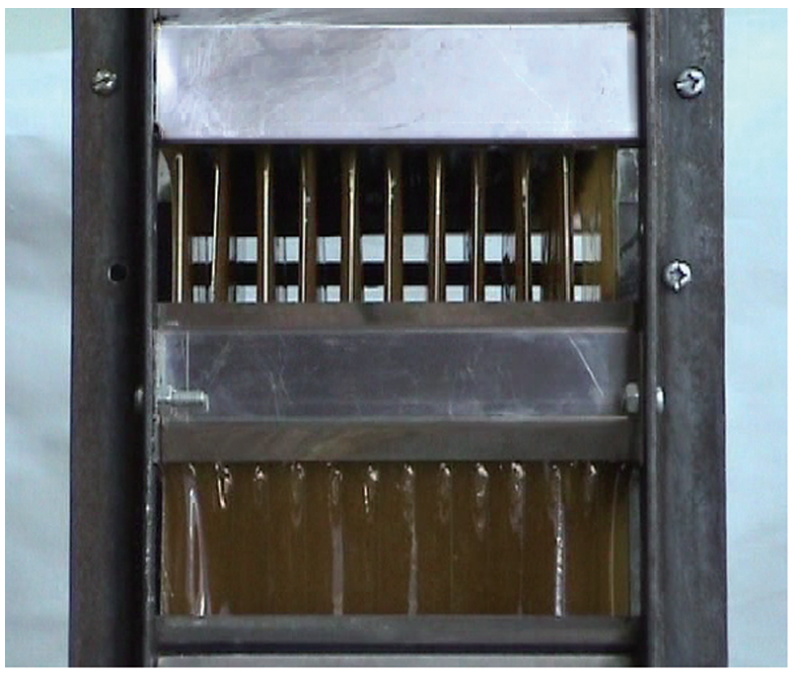

Fig. 2 Falling film forming in GFFT

condition of flow. The aqueous solutions of corn syrup with various concentrations (viscosity ranging from 1.5 to $210 \mathrm{~Pa} \cdot \mathrm{s}$ ) were chosen as experimental media, and the viscosities were measured by NDJ-4 rotational viscometer (Brookfield-type, Shenzhen Sanuo Electronic Instrument Co., Ltd.). Several model cores with different structural parameters were used to adapt to different viscous fluids.

The corn syrup solution with a certain viscosity was fed into the distributor by screw pump, then flowed down through the model core and fell into the tank for cycling. The flow rate was regulated by the frequency of the motor of the pump. The film was formed on the flow-guide combs between layers. The bubbles naturally generated on surface of falling syrup film were used as tracer elements, and the surface velocity field on falling film was measured by particle image velocimetry (PIV) technique (flow map system, Dantec Dynamics A/S). The map pairs of images were then analyzed by Flow Manager 4.5 to obtain two-dimensional velocity vectors at each point on falling film. Moreover, the thickness of falling film was also recorded by video technique.

The residence time distribution (RTD) had been tested by pulse method, and the response could be deduced from the picture recorded at outlet port by image gray processing. Red-brown syrup colored with iodine was used as pulse tracer, which was found to have linear relationship between the concentration of iodine $C_{\mathrm{I} 2}$ and gray-scale value of image when $C_{\mathrm{I} 2}$ is less than 0.005 $\mathrm{mol} / \mathrm{L}$ in our preliminary experiment.

The PET polycondensation processes in GFFT ran on the same setup and procedure as that in the early work (Xi et al., 2007). Three GFFT cores with different structural parameters were designed for different inlet PET polymers with DP of 34,62 , and 100 respectively. 


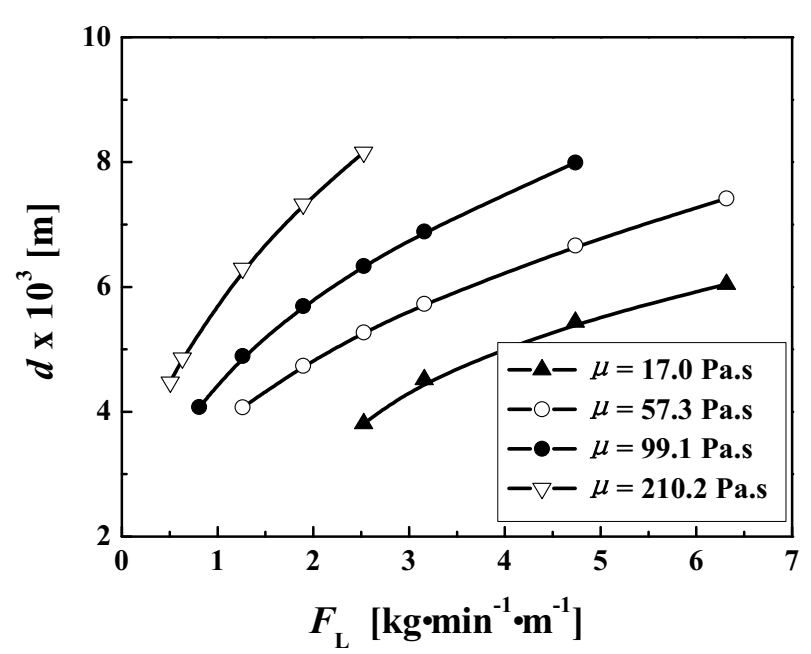

Fig. 3 Effect of flow rate on thickness of falling film for different viscous fluids, $d_{\mathrm{g}}=0.014 \mathrm{~m}$

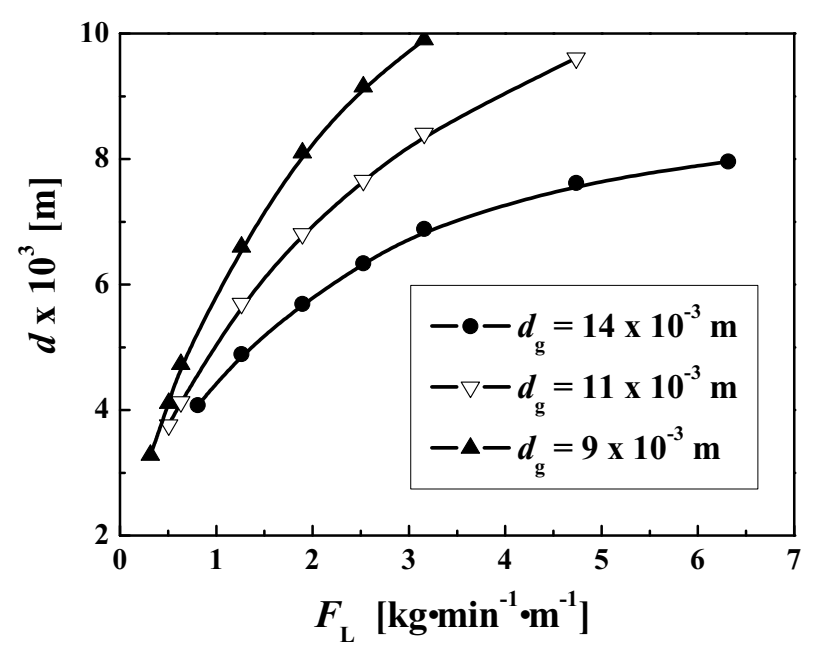

Fig. 4 Effect of core structural parameters on thickness of falling film, $\mu=99.1 \mathrm{~Pa} \cdot \mathrm{s}$

\section{Results and Discussion}

\subsection{Characteristics of falling film}

From Figure 2, it is observed that thin falling film with double free surfaces could be generated. The thickness of falling film $d$ increases with flow rate per width of comb $F_{\mathrm{L}}$ as well as fluid viscosity, see Figure 3. It also can be regulated by core structural parameters, including the width of grating slits and the gap size among comb sticks $d_{\mathrm{g}}$, especially the latter, see Figure 4. Since holdup of GFFT has direct relationship with thickness of falling film on combs and liquid level over layers, the residence time along axial GFFT could be tuned by core structural parameters.

The typical surface velocity field on falling film is shown in Figure 5. The surface velocity field is rather even other than the marginal parts. As the flow of falling

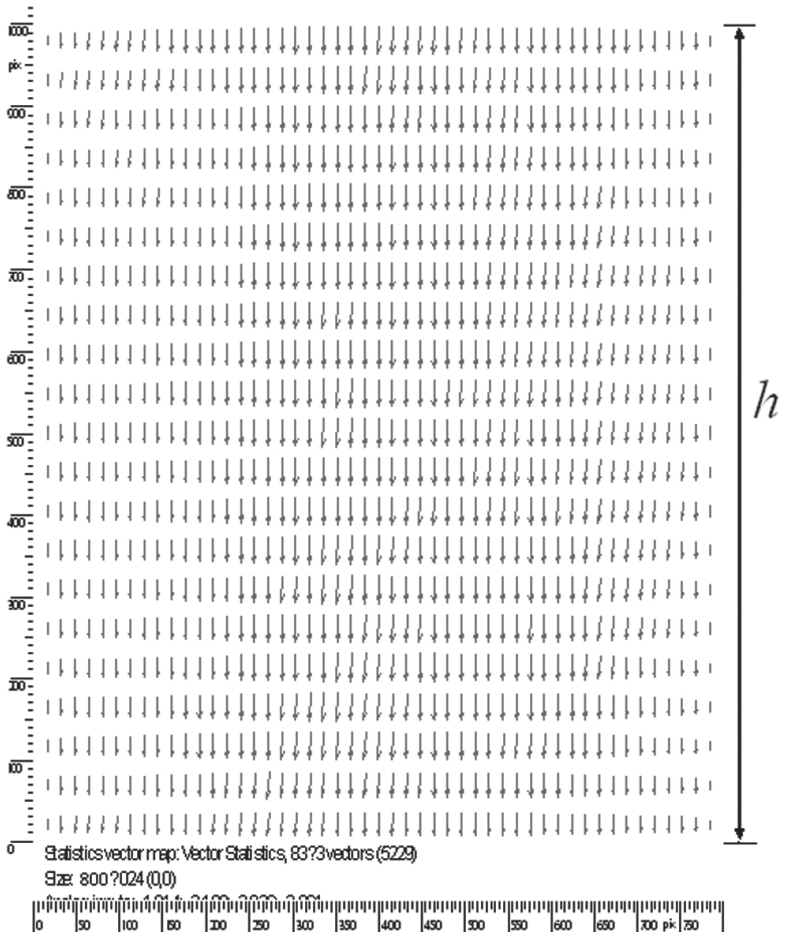

Fig. 5 Surface velocity field on falling film measured by PIV technique

film is in steady state, the surface area generated is equal to the area disappeared all the time. So if the surface renewal frequency $\gamma$ of falling film is defined as the ratio of the area generated to be exposed to vapor phase in unit time to the area of falling film supported by comb, it would be expressed as following Eq. (1).

$$
\gamma=\frac{S_{\gamma}}{S_{F}}=\frac{2 \cdot u_{h} \cdot L}{2 \cdot h \cdot L}=\frac{u_{h}}{h}
$$

where $2 \cdot u_{h} \cdot L$ is the surface area disappeared for single film in unit time, $h$ is the height of falling film, $L$ is the width of film.

And the specific interfacial area of single falling film can be directly counted out as following Eq. (2).

$$
S_{\mathrm{W}}=\frac{S_{\gamma}}{F_{\mathrm{L}} \cdot L}=\frac{2 \cdot \gamma \cdot L \cdot h}{F_{\mathrm{L}} \cdot L}=\frac{2 \cdot \gamma \cdot h}{F_{\mathrm{L}}}
$$

The experimental results show that the surface renewal frequency increases linearly with the flow rate and is inversely proportional to $\mu^{0.38}$. Moreover, it can be intensified with larger gaps of comb sticks especially under high flow rate, see Figures 6 and 7. The surface renewal frequency usually varies over a wide range with viscosity and structural parameters, which means that the appropriate structure should be devised to ensure film renewal for different fluid viscosity. Only for very high viscosity and at low flow rate, the surface renewal contributed by fluid re-mixing and falling film re-generating at every layer, which is caused by the perpendicular grids between adjacent layers, becomes more important. 


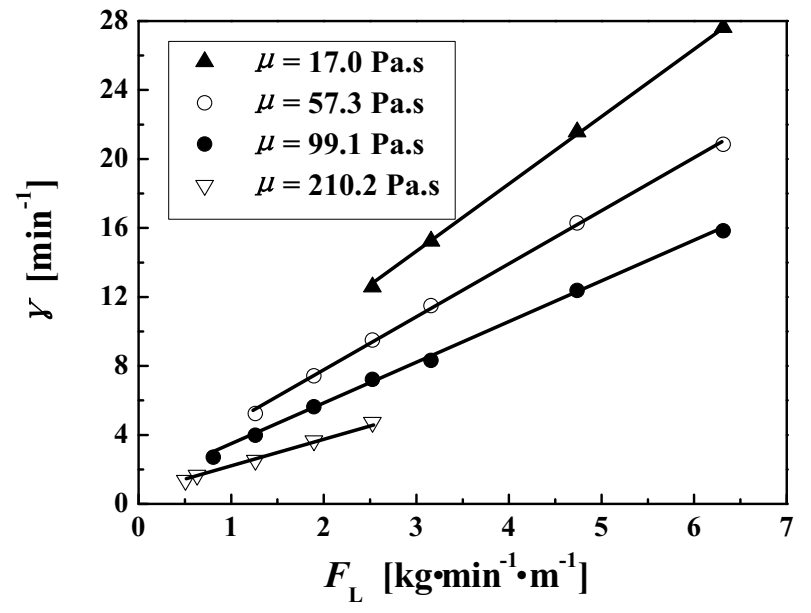

Fig. 6 Effect of flow rate on surface renewal frequency of falling film for different viscous fluids, $d_{\mathrm{g}}=0.014$ $\mathrm{mm}$

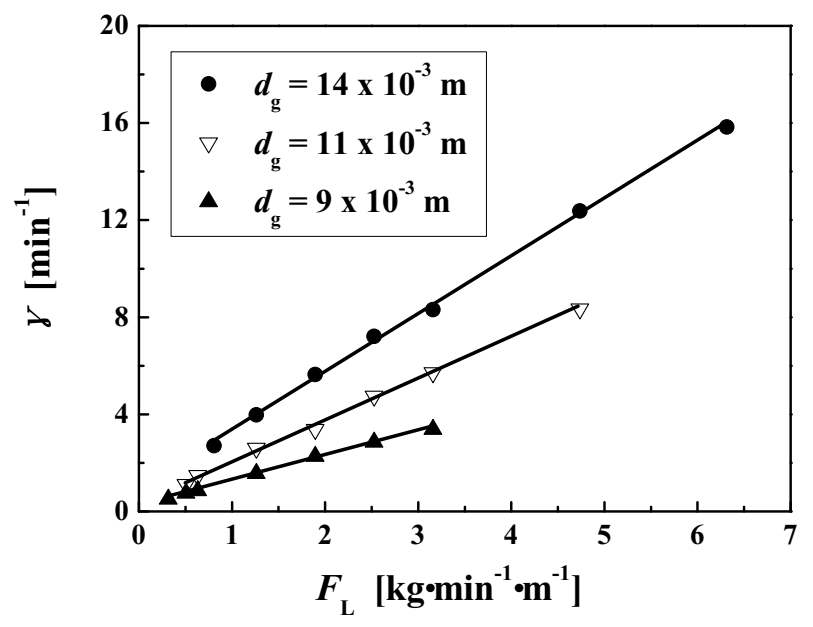

Fig. 7 Effect of core structural parameters on surface renewal frequency of falling film, $\mu=99.1 \mathrm{~Pa} \cdot \mathrm{s}$

\subsection{Fluid flow}

Figure 8 shows the typical experimental result of dimensionless RTD density function $E(\theta)$ after multilayers. The average residence time deduced from RTD is consistent with that calculated from holdup. The equivalent number of perfectly mixed tanks in series can be obtained by the reciprocal of variance of $E(\theta)$, expressed by $m$. For the same total height of core, the calculated $m$ is much more than 20 for either 2 pairs of perpendicular layers or 3 pairs of perpendicular layers. The original concept is that the fluid flowing through GFFT remixes at every layer, and the fluid flow would approach to plug flow after enough layers of core. Measurements have proved that the whole GFFT behaves as PFR due to little back-mixing in down flowing stream, irrespective of the number of layers.
Table 1 PET polycondensation processes in pilot-scale GFFT

\begin{tabular}{lccc}
\hline \multicolumn{1}{c}{ Process No. } & Process 1 & Process 2 & Process 3 \\
\hline Capacity $\left[\mathrm{kg} \cdot \mathrm{h}^{-1}\right.$ ] & 10 & 10 & 10 \\
Height $[\mathrm{m}]$ & 4 & 3 & 3 \\
Number of layers & 62 & 20 & 20 \\
Pressure [Pa] & $100-160$ & $180-200$ & $180-220$ \\
Temperature [K] & 558 & 553 & 553 \\
Residence time [min] & $\sim 30$ & $\sim 20$ & $\sim 20$ \\
DP of inlet PET & 62 & 34 & 100 \\
DP of outlet PET & $147-172$ & $78-89$ & $130-138$ \\
\hline
\end{tabular}

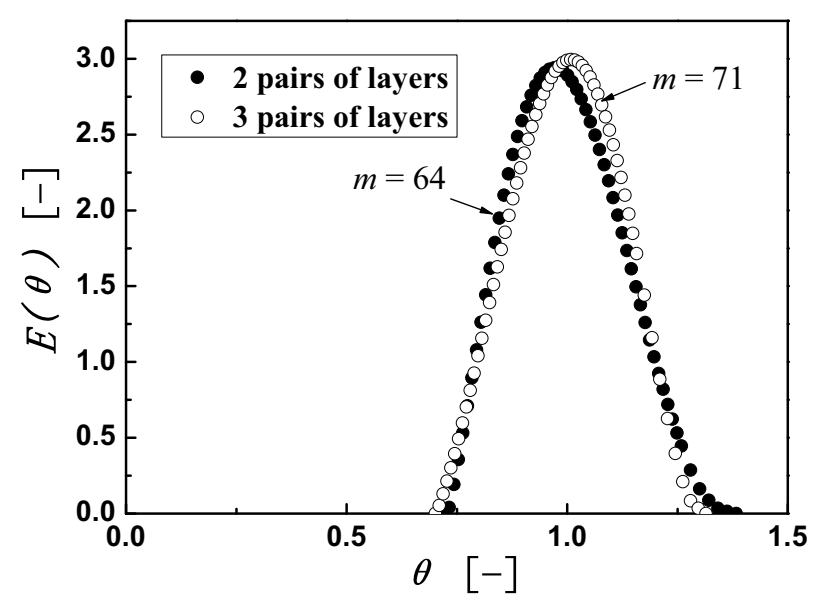

Fig. 8 Dimensionless RTD density function curve of different GFFT cores

\subsection{PET polycondensation in pilot-scale GFFT}

Several PET melt polycondensation processes have been realized successfully in GFFT, see Table 1. Within shorter residence time, the DP could be lifted up under conventional temperature and vacuum. The maximum DP of outlet PET polymer could be up to 172, which implied that traditional solid PET polycondensation process could be covered. So the GFFT is an efficient melt polycondensation reactor, manufacturing not only routine fibergrade PET product but also PET with quite high molecular weight.

\section{Modeling of Continuous PET Polycondensation Process in GFFT}

In the PET synthesis process, several functional end groups are present, including the hydroxyl group, carboxyl group, methyl ester group and diethylene glycol group. These functional end groups react with each other and reaction kinetics become quite complicated (Yoon and Park, 1994; Rieckmann and Völker, 2001). However, only the main polycondensation reaction, chain growth reaction, will be considered in our model and the focus will be given on the polymer DP development along re- 
actor axis. The chain growth reaction (Ravindranath and Mashelkar, 1982) can be expressed by Eq. (3).

$$
\mathrm{Ce}+\mathrm{Ce} \rightleftharpoons \mathrm{Z}+\mathrm{g}
$$

where Ce represents the end groups including hydroxyl end groups $-\mathrm{OH}$ and carboxyl end groups $-\mathrm{COOH}, \mathrm{Z}$ denotes diester groups, and $\mathrm{g}$ is small molecules including ethylene glycol and water.

Since PET chain growth reaction is equilibrium controlled (Gupta and Kumar, 1987), if the small molecule is removed in time, the concentration of end groups would decrease while DP would increase, and the chain growth reaction would go forward and reach new reaction equilibrium. In respect that GFFT has huge specific interfacial area and all melts are in the state of thin film exposing to high vacuum, it may be assumed that the process could reach pseudo-steady state between the reaction and masstransfer,

$$
N=k\left([\mathrm{Ce}]^{2}-\frac{4[\mathrm{Z}][\mathrm{g}]}{K}\right) W=K_{\mathrm{L}} S\left([\mathrm{~g}]-[\mathrm{g}]^{*}\right)
$$

From above equation, we can deduce an expression for [g].

$$
[\mathrm{g}]=\frac{k \cdot W \cdot[\mathrm{Ce}]^{2}+K_{\mathrm{L}} \cdot S \cdot[\mathrm{g}]^{*}}{K_{\mathrm{L}} \cdot S+\frac{4 \cdot k \cdot W}{K} \cdot[\mathrm{Z}]}
$$

If an overall apparent rate of PET melts polycondensation process is defined by the expression,

$$
\frac{\mathrm{d}[\mathrm{g}]}{\mathrm{d} t}=k_{\mathrm{T}} \cdot\left([\mathrm{Ce}]^{2}-\frac{4[\mathrm{Z}] \cdot[\mathrm{g}]^{*}}{K}\right)
$$

we can infer that,

$$
\frac{1}{k_{\mathrm{T}}}=\frac{1}{k}+\frac{[\mathrm{Z}] / K}{K_{\mathrm{L}} \cdot s_{\mathrm{W}}}
$$

where $K_{\mathrm{L}} \cdot S_{\mathrm{w}}$ is a composition-dependent volumetric mass-transfer coefficient, which is similar to diffusion coefficient as a function of DP or concentration of small molecules in some models (Pell and Davis, 1973; Rieckmann and Völker, 2001), $k$ is the rate constant of forward reaction and $K$ reaction equilibrium constant, and $[\mathrm{Ce}],[\mathrm{Z}],[\mathrm{g}]^{*}$ represent the concentration of PET end groups, diester groups, small molecules at the gas-liquid interface respectively. The total end groups' concentration $[\mathrm{Ce}]$ can be calculated from $M_{\mathrm{n}}$ (Yamada and Imamura, 1989).

From stoichimetric coefficients of chain growth reaction, see Eq. (3), it is easy to derive

$$
r=-\frac{\mathrm{d}[\mathrm{Ce}]}{\mathrm{d} t}=2 \times \frac{\mathrm{d}[\mathrm{g}]}{\mathrm{d} t}
$$

The parameters in above model have been estimated on the basis of experimental data of PET melt polycondensation process in thin film reactors with different film thicknesses in our previous works (Zhao, 1999; Zhao et al., 2002).
Since GFFT reactor behaves as PFR, for any infinitesimal section, the mass balance equation for end groups can be written as

$$
F \cdot \mathrm{d}[\mathrm{Ce}]=(-r) \cdot \mathrm{d} W
$$

where $F$ is the mass flow rate of polymer melt, which is almost constant because of very small amount of volatiles being removed during later PET polycondensation process, and $\mathrm{d} W$ is liquid holdup embodied in this infinitesimal section. In GFFT, polymer melt flows through multilayers grids to provide large gas-liquid interfacial area for the removal of small molecules, and the DP as well as fluid viscosity, direct related to $M_{\mathrm{n}}$ (Lee et al., 1992), increases continuously with polycondensation proceeding. The core structural parameters must be changed to adapt to different fluid viscosity. Consequently, liquid holdup, surface renewal overall and the apparent rate of PET melt polycondensation process will change along with axial position (the height of the core, $h$ ). Integrating with film forming and renewal in sections with different core structural parameters, from inlet to outlet, axial, $[\mathrm{Ce}]$ and DP distribution can be calculated out by a fourth order Runge-Kutta method. Figures 9-11 displayed the simulation results of three PET melt polycondensation processes in GFFT shown in Table 1. Under the conditions labeled in Figures, the experimental outlet DP for three processes is 170, 89 and 138 respectively, and the calculated is 162,85 and 135 correspondingly; the relative error between them is within $4.7 \%$. So the simulation work could fit experimental data well and provide some valuable information for the optimization of reactor design, that is, how the structural parameters of GFFT along the axial direction should be tuned to match the progress of PET polycondensation process.

\section{Conclusions}

Owing to its excellent performances of fluid flow and film forming, GFFT can be regarded as an efficient apparatus for PET melt polycondensation process to manufacture polymers with high molecular weight. With PET polcondensation proceeding, the axial DP as well as fluid viscosity rises continuously, and the core structural parameters must be set to adapt to the fluid viscosity.

The characteristics of falling film and fluid flow in GFFT have been investigated using the aqueous solutions of corn syrup with the viscosity range of 1.5-210 $\mathrm{Pa} \cdot \mathrm{s}$. as model viscous fluids. The fluid flow in GFFT approaches to ideal plug flow, and the residence time along GFFT axial position is adjustable. The surface renewal of falling films is mainly contributed by its surface flowing and varies over a wide range with different structures and operation conditions. It is important to devise appropriate structures to intensify the film renewal for mass transfer especially for high viscous fluids.

A realistic model has been developed for the continuous PET melt polycondensation process in GFFT. In the 


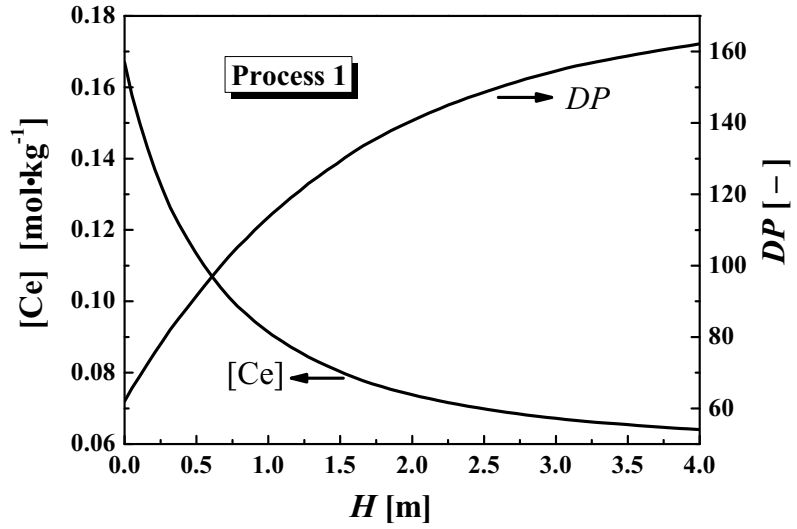

Fig. $9[\mathrm{Ce}]$ and DP axial distribution for process $1, T=$ $558.1 \mathrm{~K}, P=100 \mathrm{~Pa}, t=30 \mathrm{~min}$

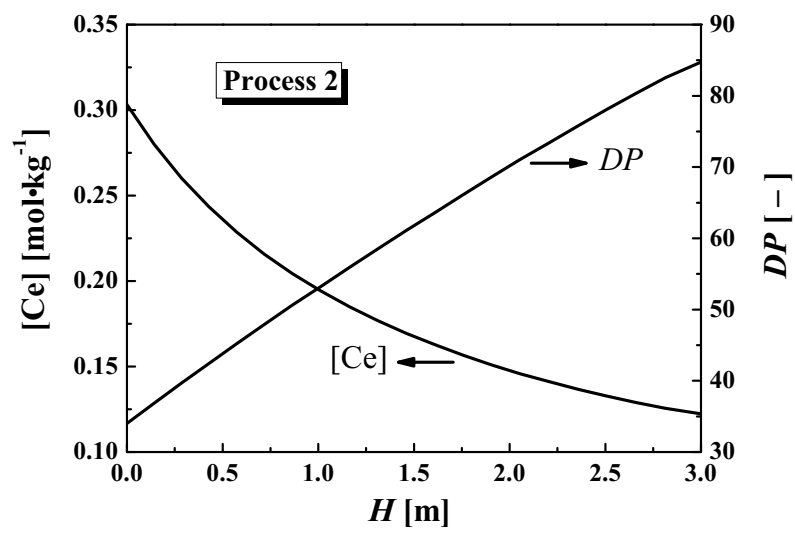

Fig. $10[\mathrm{Ce}]$ and DP axial distribution for process $2, T=$ 553.1 K, $P=180 \mathrm{~Pa}, t=20 \mathrm{~min}$

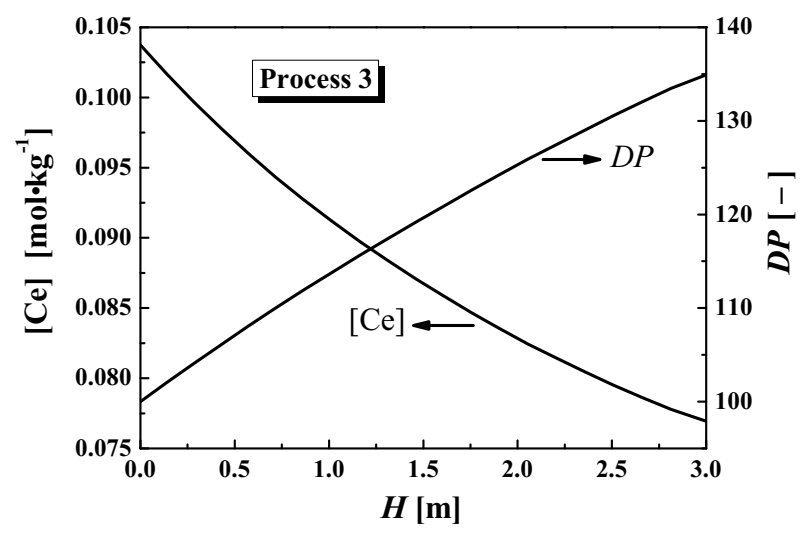

Fig. $11[\mathrm{Ce}]$ and DP axial distribution for process $3, T=$ $553.1 \mathrm{~K}, P=180 \mathrm{~Pa}, t=20 \mathrm{~min}$

model, an overall apparent rate is proposed to describe PET melt polycondensation process in thin film. The developed model was verified by experimental data of PET melt polycondensation process in pilot-scale GFFT. The calculated axial DP changing trend would be useful for optimum reactor design.

\section{Acknowledgments}

The authors appreciate the financial support from the Innovative Research Team in University (IRT0721) and the 111 Project of China (B08021).

\begin{tabular}{|c|c|c|}
\hline \multicolumn{2}{|c|}{ Nomenclature } & \multirow[b]{2}{*}{ concentration of PET end group } \\
\hline$[\mathrm{Ce}]$ & $=$ & \\
\hline$C_{\mathrm{I} 2}$ & $=$ & concentration of iodine \\
\hline$D P$ & $=$ & degree of polymerization \\
\hline$d$ & $=$ & film thickness \\
\hline$d_{\mathrm{g}}$ & $=$ & gap size among comb sticks \\
\hline$E(\theta)$ & $=$ & $\begin{array}{l}\text { dimensionless residence time distribution density } \\
\text { function }\end{array}$ \\
\hline$F$ & $=$ & mass flow rate \\
\hline$F_{\mathrm{L}}$ & $=$ & $\begin{array}{l}\text { mass flow rate of single film with unit width of comb } \\
\qquad\left[\mathrm{kg} \cdot \mathrm{min}^{-1} \cdot \mathrm{m}^{-1}\right]\end{array}$ \\
\hline [g] & $=$ & concentration of small molecules \\
\hline$[\mathrm{g}]^{*}$ & $=$ & interfacial concentration of small molecules \\
\hline & & {$\left[\mathrm{mol} \cdot \mathrm{kg}^{-1}\right]$} \\
\hline$H$ & $=$ & axial height of the core \\
\hline$h$ & $=$ & height of falling film \\
\hline$K$ & $=$ & equilibrium constant \\
\hline$K_{\mathrm{L}}$ & $=$ & mass transfer coefficient \\
\hline$K_{\mathrm{L}}$. & $=$ & volumetric mass transfer coefficient \\
\hline & & {$\left[\mathrm{m}^{3} \mathrm{~kg}^{-1} \min ^{-1}\right]$} \\
\hline$k$ & $=$ & rate constant of forward reaction $\left[\mathrm{kg} \cdot \mathrm{mol}^{-1} \cdot \mathrm{min}^{-1}\right]$ \\
\hline$k_{\mathrm{T}}$ & $=$ & overall apparent rate constant \\
\hline$L$ & $=$ & width of comb \\
\hline$M_{\mathrm{n}}$ & $=$ & number-average molecular weight of polymer [-] \\
\hline$m$ & $=$ & number of perfectly mixed tanks in series \\
\hline$N$ & $=$ & mass flux of small molecules in unit time \\
\hline & & {$\left[\mathrm{mol} \cdot \mathrm{min}^{-1}\right]$} \\
\hline$n$ & $=$ & number of layers \\
\hline$P$ & $=$ & pressure \\
\hline$r$ & $=$ & reaction rate of end groups \\
\hline$S$ & $=$ & area for masstransfer \\
\hline$S_{\mathrm{F}}$ & $=$ & area of falling film supported by comb \\
\hline$S_{\mathrm{W}}$ & $=$ & interfacial area per unit weight of polymer \\
\hline & & {$\left[\mathrm{m}^{2} \cdot \mathrm{kg}^{-1}\right]$} \\
\hline$S_{\gamma}$ & $=$ & $\begin{array}{l}\text { area generated to be exposed to vapor phase in unit } \\
\text { time } \\
\left.\qquad \mathrm{m}^{2} \cdot \mathrm{min}^{-1}\right]\end{array}$ \\
\hline$T$ & $=$ & temperature \\
\hline$t$ & $=$ & residence time \\
\hline$u$ & $=$ & surface velocity of falling film \\
\hline$W$ & $=$ & liquid holdup \\
\hline$[\mathrm{Z}]$ & $=$ & concentration of diester groups \\
\hline$\theta$ & $=$ & dimensionless time \\
\hline$\gamma$ & $=$ & film renewal frequency \\
\hline & $=$ & viscosity of fluids \\
\hline
\end{tabular}

\section{Literature Cited}

Crawford, J. E., R. W. Edwards, E. D. Hease and W. C. L. Wu; "Apparatus for Polymerizing Liquids," U.S. Patent 3,619,145 (1971)

Gupta, S. K. and A. Kumar; Reaction Engineering of Step Growth Polymerization, pp. 255, Plenum Press, New York, U.S.A. (1987)

Hachiya, M., K. Hiratsuka and H. Fukumori; "Thin Film Continuous Evaporating Apparatus," U.S. Patent 3,532,151 (1970)

Kilpatrick, L. L.; “Polymer Finisher Apparatus,” U.S. Patent 3,248,180 (1966)

Lee, K. J., D. Y. Moon, O. O. Park and Y. S. Kange; "Diffusion of Ethylene Glycol Accompanied by Reactions in Poly(Ethylene Terephthalate) Melts," J. Polym. Sci. Part B: Polym. Phys., 30, 707-716 (1992) 
Lester, L. K. and N. C. Grifon; "Polymerfinishing Apparatus," U.S. Patent 3,526,484 (1970)

Liu, Z.-Y.; "A Grid Tower for Polycondensation of Polyester" (in Chinese), Patent CN1,199,651 (1998)

Ogata, K., K. Kazama, S. Suzuki and Y. Fukumori; "Novel Process for the Preparation of Polyester," U.S. Patent 3,442,868 (1969)

Pell, T. M. and T. G. Davis; "Diffusion and Reaction in Polyester Melts," J. Polym. Sci. Part B: Polym. Phys., 11, 1671-1682 (1973)

Ravindranath, K. and R. A. Mashelkar; "Modeling of Poly(Ethylene Terephthalate) Reactors: VI. A Continuous Process for Final Stages of Polycondensation," Polym. Eng. Sci., 22, 628-636 (1982)

Ravindranath, K. and R. A. Mashelkar; "Finishing Stages of PET Synthesis: A Comprehensive Model," AIChE J., 30, 415-422 (1984)

Rieckmann, Th. and S. Völker; "Micro-Kinetics and Mass Transfer in Poly(Ethylene Terephthalate) Synthesis," Chem. Eng. Sci., 56, 945953 (2001)

Wilhelm, F., H.-G. Witt and L. Hoelting; "Apparatus for Processing High Viscosity Materials," U.S. Patent 5,055,273 (1991)

Xi, Z.-H., L. Zhao and Z.-Y. Liu; "New Falling Film Reactor for Melt
Polycondensation Process," Macromol. Symp., 259, 10-16 (2007) Yamada, T. and Y. Imamura; "Simulation of Continuous Direct Esterification Process between Terephthalic Acid and Ethylene Glycol," Polym. Plast. Technol. Eng., 28, 811-876 (1989)

Yokoyama, H. and K. Fujimoto; "Process for Producing Polyalkylene Terephthalate, Process for Producing Polyalkylene Terephthalate Molding and Polyalkylene Terephthalate Molding," Patent WO 2005035620 (2005)

Yoon, K. H. and O. O. Park; "Analysis of a Reactor with Surface Renewal for Poly(Ethylene Terephthalate) Synthesis," Polym. Eng. Sci., 34, 190-200 (1994)

Zhao, L.; "Reaction and Devolatilization in PET Polycondensation Process" (in Chinese), Ph.D. Thesis, East China University of Science and Technology, China (1999)

Zhao, L., Z.-N. Zhu, Y.-C. Dai and G.-C. Dai; "Reaction and Mass Transfer in Poly(Ethylene Terephthalate) Melt Polycondensation Process," MS\#0063, 17th International Symposia on Chemical Reaction Engineering, Hong Kong, China (2002) 